Research Article

\title{
Jiawei Foshou San Induces Apoptosis in Ectopic Endometrium Based on Systems Pharmacology, Molecular Docking, and Experimental Evidence
}

\author{
Jiahui Wei, ${ }^{1,2,3,4}$ Binxin Zhao, ${ }^{1,2,3,4}$ Chengling Zhang, ${ }^{1,2,3,4}$ Bingbing Shen, ${ }^{5}$ Ying Zhang, ${ }^{1,2,3,4}$ \\ Changxi $L i,{ }^{6}$ and Yi Chen $\mathbb{D}^{1,2,3,4}$ \\ ${ }^{1}$ College of Pharmaceutical Sciences \& Chinese Medicine, Southwest University, Chongqing, China \\ ${ }^{2}$ Chongqing Key Laboratory of New Drug Screening from Traditional Chinese Medicine, Chongqing, China \\ ${ }^{3}$ Pharmacology of Chinese Materia Medica-the Key Discipline Constructed by the State Administration of Traditional , \\ Chinese Medicine, Chongqing, China \\ ${ }^{4}$ National Demonstration Center for Experimental Pharmacy Education (Southwest University), Chongqing, China \\ ${ }^{5}$ The First Affiliated Hospital to Army Medical University, Chongqing 400038, China \\ ${ }^{6}$ Department of Anesthesia, Chongqing Public Health Medical Center, Chongqing, China
}

Correspondence should be addressed to Yi Chen; rachelcy@swu.edu.cn

Received 13 May 2019; Revised 9 August 2019; Accepted 19 September 2019; Published 27 October 2019

Academic Editor: Olumayokun A. Olajide

Copyright (c) 2019 Jiahui Wei et al. This is an open access article distributed under the Creative Commons Attribution License, which permits unrestricted use, distribution, and reproduction in any medium, provided the original work is properly cited.

Foshou San is a typical gynaecological formula with wild usage in traditional Chinese medicine. Jiawei Foshou San (JFS) is a novel ingredient prescription from Foshou San with antiendometriosis effect in unclear mechanisms. To uncover the potential application and proapoptotic mechanisms of JFS, JFS ingredient-drug target-disease networks, GO enrichment, and pathway analysis were established for potential application prediction. Molecular docking and validation in vivo were investigated by the proapoptotic mechanisms of JFS. In this study, 99 common targets were related to 108 diseases. 484 biological processes, 44 cell components, 59 molecular functions, and 37 pathways were significantly identified in GO enrichment and pathway analysis. In molecular docking, ligustrazine showed binding activity with Bcl-2, Bax, caspase-9, caspase-3, and PARP. In vivo, JFS elevated the shrink rate of ectopic endometrium, by suppressing E2 and PROG. An in-depth study showed that apoptosis was activated through diminishing Bcl-2, rising Bax and Bad, and expressing more caspase- 3 and caspase- 9 using JFS. JFS promoted the protein level of cleaved-PARP. In brief, JFS might be applied for various diseases through multiple targets and pathways, especially endometriosis by Bcl-2 pathway. These findings reveal the potential application for further evaluation and uncover the proapoptotic mechanism of JFS.

\section{Introduction}

Blood stasis syndrome in traditional Chinese medicine (TCM) is considered appearing in various chronic diseases, such as cardiocerebrovascular diseases, gynaecological diseases, tumor, and infectious diseases [1-3]. Ниохие Ниауи recipe is regarded to ameliorate the blood stasis syndrome through activating blood and dissolving stasis in TCM $[1,4]$. Foshou San formula is a classic Hиохиe Hиауu recipe, utilized extensively in gynaecological diseases, cardiocerebrovascular diseases, and hepatobiliary disease [5]. Two main active ingredients of Foshou San, ligustrazine and ferulic acid, are coordinated with tetrahydropalmatine to form Jiawei Foshou San (JFS). Our previous research has found that JFS has a good therapeutic effect on endometriosis (EMS), including reducing the inflammatory response and antimetastasis $[3,6]$. However, the proapoptotic mechanism of JFS has not been measured in EMS. At the same time, it is worth to detect the potential application of JFS on disease belonging to blood stasis syndrome. 
Systems pharmacology is a novel research field combined with pharmacology and systems biology-based technologies. Multicomponent and multitarget therapeutics and potential treatment of complex diseases are usually considered as the characteristics of TCM formulas. Thus, application of systems pharmacology in TCM will be helpful to uncover the interactions between components, targets, and pathways $[7,8]$. Because not all targets are efficient therapeutic targets, systems pharmacology combined with FDA drug target database might be an efficient and promising way to broaden the potential therapeutic range of TCM [9].

EMS, one of the high-incidence gynaecological diseases, is defined as the presence of the active endometrial tissues at extrauterine sites $[10,11]$. Currently, the pathogenesis of EMS is still unclear. Apoptosis is a physiologic process of programmed cell degeneration and necrosis under the action of apoptotic stimuli. It has been shown previously that abnormal apoptosis of endometrial cells is closely related to the occurrence and development of EMS [12, 13].

In this study, systems pharmacology was employed to establish JFS target-drug target and common target-disease networks. Then, the common targets were analysed for GO enrichment and pathway analysis. Binding activity of components and apoptosis-related targets was predicted by molecular docking. The proapoptosis of JFS was investigated through Bcl-2 pathway in the EMS rat model (Figure 1).

\section{Materials and Methods}

2.1. Networks Construction, GO Enrichment, and Pathway Analysis. TCMSP (http://lsp.nwu.edu.cn/tcmsp.php), SEA (http://sea.bkslab.org/), and BATMAN-TCM (http://bionet. ncpsb.org/batman-tcm/) databases were applied for potential targets of JFS compounds with the limitation of prediction score $>80$ and $P>0.05$ [14]. DrugBank (https:// www.drugbank.ca/drugs) and TTD (https://db.idrblab.org/ $\mathrm{ttd} /$ ) database were used to download pharmacological drug targets approved by the FDA. Common targets were screened by comparing JFS targets with FDA-approved drug targets. The connection between common targets and diseases was obtained from TTD and TCMSP. The diseases were classified according to $\mathrm{MeSH}$ (http://www.nlm.nih. gov/mesh/). Cytoscape 3.7 .0 (http://www.cytoscape.org/) was used to generate the JFS target-drug target and common target-disease networks. Common targets were subjected to DAVID (https://david-d.ncifcrf.gov/) for GO enrichment and pathway analysis.

2.2. Molecular Docking. In SystemsDock Website (http:// systemsdock.unit.oist.jp/iddp/home/index), molecular docking was analysed between JFS components with apoptosis-related targets. It shows certain binding activity with docking score $>4.25$, better binding activity with docking score $>5.0$, and strong binding activity with docking score $>7.0[15]$.

2.3. Reagents and Animals. The purity of ferulic acid (batch number: ZL2016061382), ligustrazine (batch number:
ZL2016030815), and tetrahydropalmatine (batch number: ZL2016051235) were 99.8\%, 99.3\%, and 98.1\%, respectively, from Nanjing Zelang Medical Technology (Nanjing, China). They were dispersed in $0.5 \%$ CMC-N with a ratio of $10: 5: 3$. Gestrinone was purchased from Zizhu Pharmaceutical Co., Ltd. (Beijing, China). Rat PROG (CK-E30608R) and E2 ELISA Kit (CK-E30580R) were purchased from Yuanye Biomart (Shanghai, China). Primers for qPCR were designed by Beijing Dingguo Changsheng Biotechnology Co., Ltd. (Beijing, China). PrimeScript ${ }^{\mathrm{TM}}$ RT Reagent Kit was supplied by TaKaRa Bio Inc (Japan). SYBR ${ }^{\mathrm{TM}}$ Green Master Mix (A25741) was obtained from Thermo Fisher Scientific (USA). TUNEL Apoptosis Detection Kit (A113-01) was provided by Vazyme (Nanjing, China). DAPI solution (C0204002) was purchased from Bioss (Beijing, China). Female Sprague Dawley rats weighing 180-220 g were supplied by Experimental Animals Institute of Chongqing Academy of Chinese Materia (Certification no. SCXK [yu] 2017-0003). They were housed in the Experimental Center, Southwest University. The study was conducted in accordance with the recommendations in the Guide for the Care and Use of Laboratory Animals of Southwest University (Approval no. 0002183). To minimize suffering, anesthesia with chloral hydrate $\left(350 \mathrm{mg} \cdot \mathrm{kg}^{-1}\right)$ was administered intraperitoneally in surgical procedure and blood collection.

2.4. EMS Rat Model and Treatment. According to the previous study, 71 estrous rats were autotransplanted of selfuterine to establish the EMS model [3]. The right uterine was divided into $5 \times 5 \mathrm{~mm}$ pieces for transplantation to the left peritoneum. Volume formula of endometrial autografts was calculated with $0.52 \times$ length $\times$ width $\times$ height by vernier caliper after 28 days [16]. Endometrial autografts, larger than $20 \mathrm{~mm}^{3}$ with surface blood, were regarded as the successful EMS models. Then, EMS rats were randomly separated into 4 groups according to the volume of endometrial autografts, EMS group with $0.5 \%$ CMC-Na, 45, 90, $180{\mathrm{mg} \cdot \mathrm{kg}^{-1} \mathrm{JFS}}^{-1}$ groups, and $0.5 \mathrm{mg} \cdot \mathrm{kg}^{-1}$ gestrinone group. After chronically administered for 28 days, the volume change rate was calculated with the formula (volume after treatment-volume pretreatment)/volume pretreatment $\times 100 \%$.

2.5. Detection of E2 and PROG in Serum by ELISA. Serum of abdominal aorta was prepared for analysis. Following ELISA kit instruction, the equilibrated plates were added with serum samples and dilution reagents. The HRPconjugated reagent was put into each well, which was covered with adhesive strips. After washing, chromogenic and stop solutions were inputted step by step. Absorbance readings at $450 \mathrm{~nm}$ were probed with ELX800 Universal Microplate Reader (Bio-Tek Instruments, USA).

2.6. TUNEL Assay. TUNEL assay was performed according to the manufacturer's protocol. In brief, after dewaxed and hydrated, the tissue sections were incubated with proteinase $\mathrm{K}$ for $20 \mathrm{~min}$ at room temperature. After incubated with equilibration buffer, sections were covered 


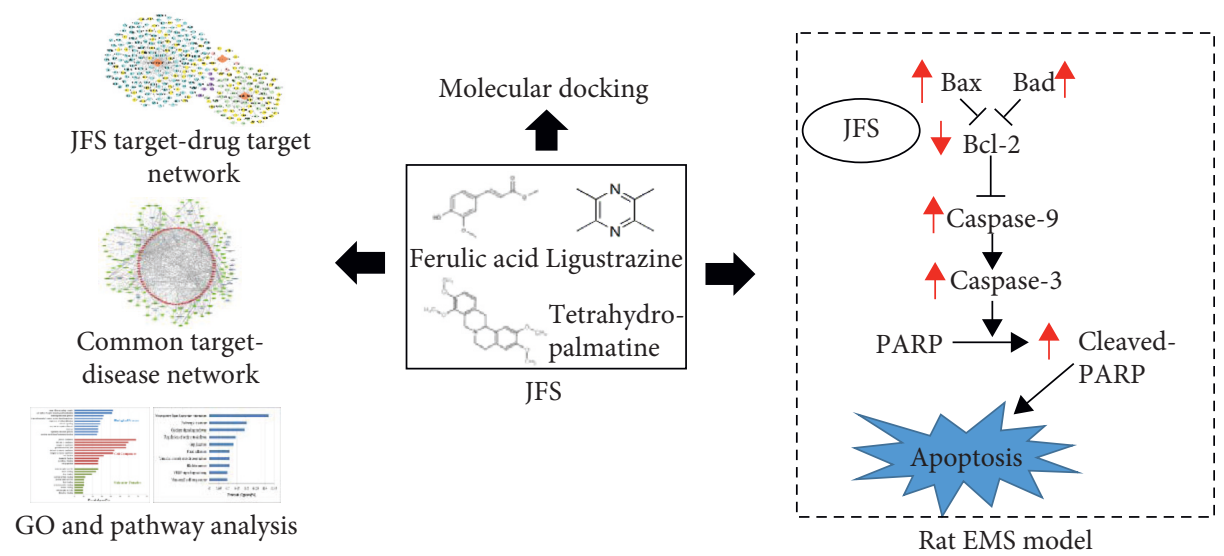

FIGURE 1: The flowchart of this study based on an integration of network pharmacology, molecular docking, and experimental evidence. JFS, Jiawei Foshou San; GO, Gene Ontology; EMS, endometriosis.

with TUNEL reaction mixture for $1 \mathrm{~h}$ at $37^{\circ} \mathrm{C}$. Finally, the sections were washed in PBS three times and counterstained with DAPI. The sections were examined and photographed under fluorescence microscopy (DFC310 FX, Leica, Germany). Apoptosis index $(\mathrm{AI})=$ apoptotic cell number/total cell number $\times 100 \%$ [17].

2.7. Real-Time PCR. Total RNA was extracted from ectopic endometrium using TRIzol reagent (Invitrogen, CA, USA) following the process described previously [18]. The synthesis of cDNA was performed with PrimeScript ${ }^{\mathrm{TM}} \mathrm{RT}$ Reagent Kit. The mRNA levels were detected using SYBR ${ }^{\mathrm{TM}}$ Green Master Mix with CFX96 Real-Time System (Bio-Rad, USA). The relative expression of target genes was calculated by the $2-\Delta \Delta \mathrm{CT}$ method using primer sequences (Table 1 ). The control reference gene was GAPDH.

2.8. Western Blot Analysis. Protein lysates of ectopic endometrium were separated by 10 or $12 \%$ SDS-PAGE and transferred to polyvinylidene difluoride membranes (Millipore, USA). The membranes were blocked with 5\% skim milk in TBST for 2 hours. The membranes were incubated overnight at $4^{\circ} \mathrm{C}$ with rabbit anti-Bcl-2, rabbit anti-Bax (1: 500 dilution; Proteintech Biotechnology, Wuhan, China), rabbit anti-PARP $(1: 250$ dilution; Biosynthesis Biotechnology, Beijing, China), rabbit anti-cleaved-PARP (1: 1000 dilution; Cell Signaling Technology, USA), and rabbit anti- $\beta$-actin (1:5000 dilution; Proteintech Biotechnology, Wuhan, China). After washing, the membranes were probed with HRP-conjugated goat anti-rabbit secondary antibody (1:1000 dilution; Multi Sciences, Hangzhou, China). The blots were analysed with the Tanon 5200 imaging system (Tanon, China) with $\beta$-actin as an internal control.

2.9. Statistical Analysis. All data were presented as mean \pm SD and compared by one-way ANOVA test using SPSS software (version 21). $P<0.05$ was regarded as statistically significant difference.
TABLE 1: Sequences of primers.

\begin{tabular}{lc}
\hline Primer name & Sequences $\left(5^{\prime}-3^{\prime}\right)$ \\
\hline Bcl-2-F & GGTGAACTGGGGGAGGATTG \\
Bcl-2-R & AGAGCGATGTTGTCCACCAG \\
Bax-F & AAGAAGCTGAGCGAGTGTCT \\
Bax-R & CAAAGATGGTCACTGTCTGC \\
Bad-F & CCGAAGAATGAGCGATGAAT \\
Bad-R & GATAATGCGCGTCCAACTG \\
Caspase-9-F & AGCTGGCCCAGTGTGAATAC \\
Caspase-9-R & GCTCCCACCTCAGTCAACTC \\
Caspase-3-F & TGTATGCTTACTCTACCGCACCCG \\
Caspase-3-R & GCGCAAAGTGACTGGATGAACC \\
PARP-F & CCAGCAGAAGGTCAAGAAGAC \\
PARP-R & ACCTCCATGCTGGCCTTT \\
GAPDH-F & AGACAGCCGCATCTTCTTGT \\
GAPDH-R & CTTGCCGTGGGTAGAGTCAT \\
\hline
\end{tabular}

\section{Results}

3.1. JFS Target-Drug Target Network Construction and Analysis. Through TCMSP, SEA, and BATMAN-TCM databases, there were 293 potential targets corresponding to JFS ingredients, including 10 for ligustrazine, 86 for ferulic acid, and 197 for tetrahydropalmatine (Table S1). 1215 FDAapproved drug targets were obtained from DrugBank and TTD databases (Table S2). JFS target-drug target network consisted of 290 nodes and 293 edges (Figure 2). 99 common targets were shifted from the network, 7 in ligustrazine, 44 in ferulic acid, and 54 in tetrahydropalmatine, including Bcl-2 (Table S3). Interestingly, those 6 targets were belonged to not only ferulic acid and tetrahydropalmatine targets, but also FDA-approved drug targets, such as ADRB2, CA2, F3, PTGS1, SLC6A2, and SLC6A3. It was suggested that the therapeutic properties of JFS might be based on these targets.

3.2. Common Target-Disease Network Construction and Analysis. The common target-disease network consisted of 216 nodes and 766 edges. 99 common targets were related to 108 diseases of 30 groups (Figure 3, Table S4). A large proportion of common targets were distributed in nervous 


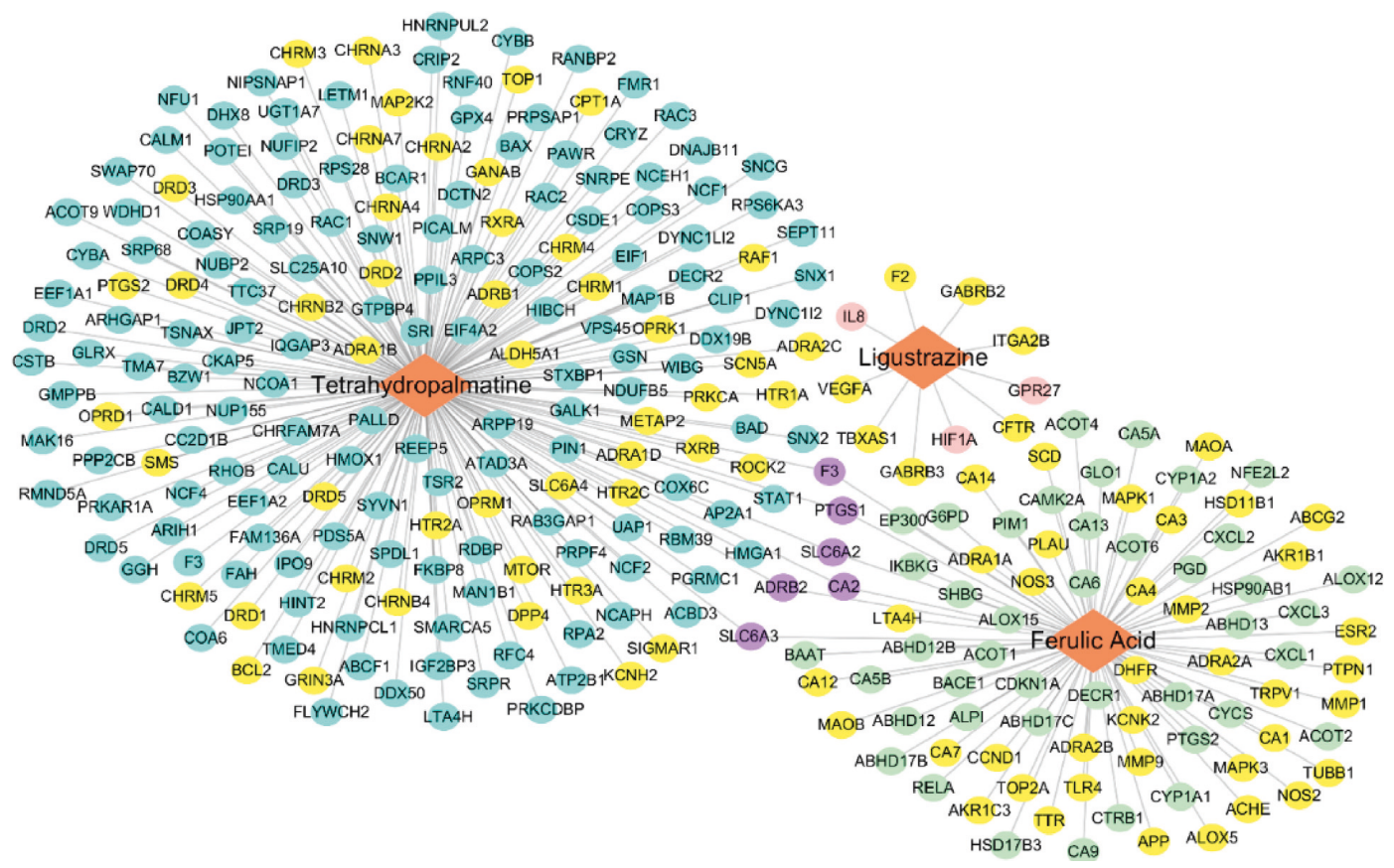

FiguRE 2: JFS target-drug target network. Blue circles, pink circles, and green circles represent the targets of tetrahydropalmatine, ligustrazine, and ferulic acid. Yellow circles represent 93 common targets between JFS targets and FDA-approved drug targets. Purple circles represent the common targets for ferulic acid, tetrahydropalmatine, and FDA-approved drug targets. JFS, Jiawei Foshou San.

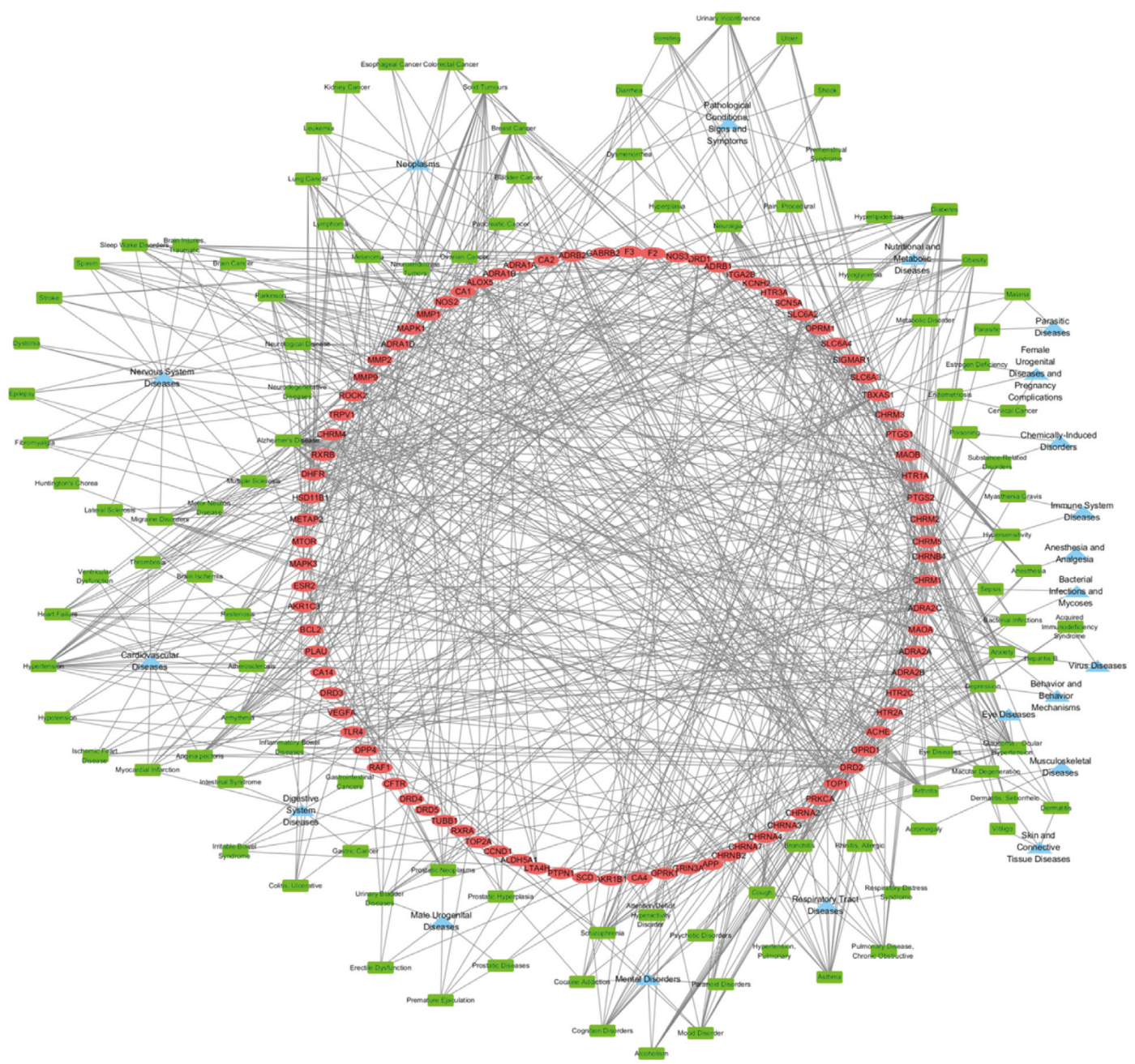

FIGURE 3: Common target-disease network. Red ellipses represent 99 common targets, and green rectangles represent diseases. 108 diseases were classified into 30 categories according to the MeSH database. MeSH, Medical Subject Headings. 


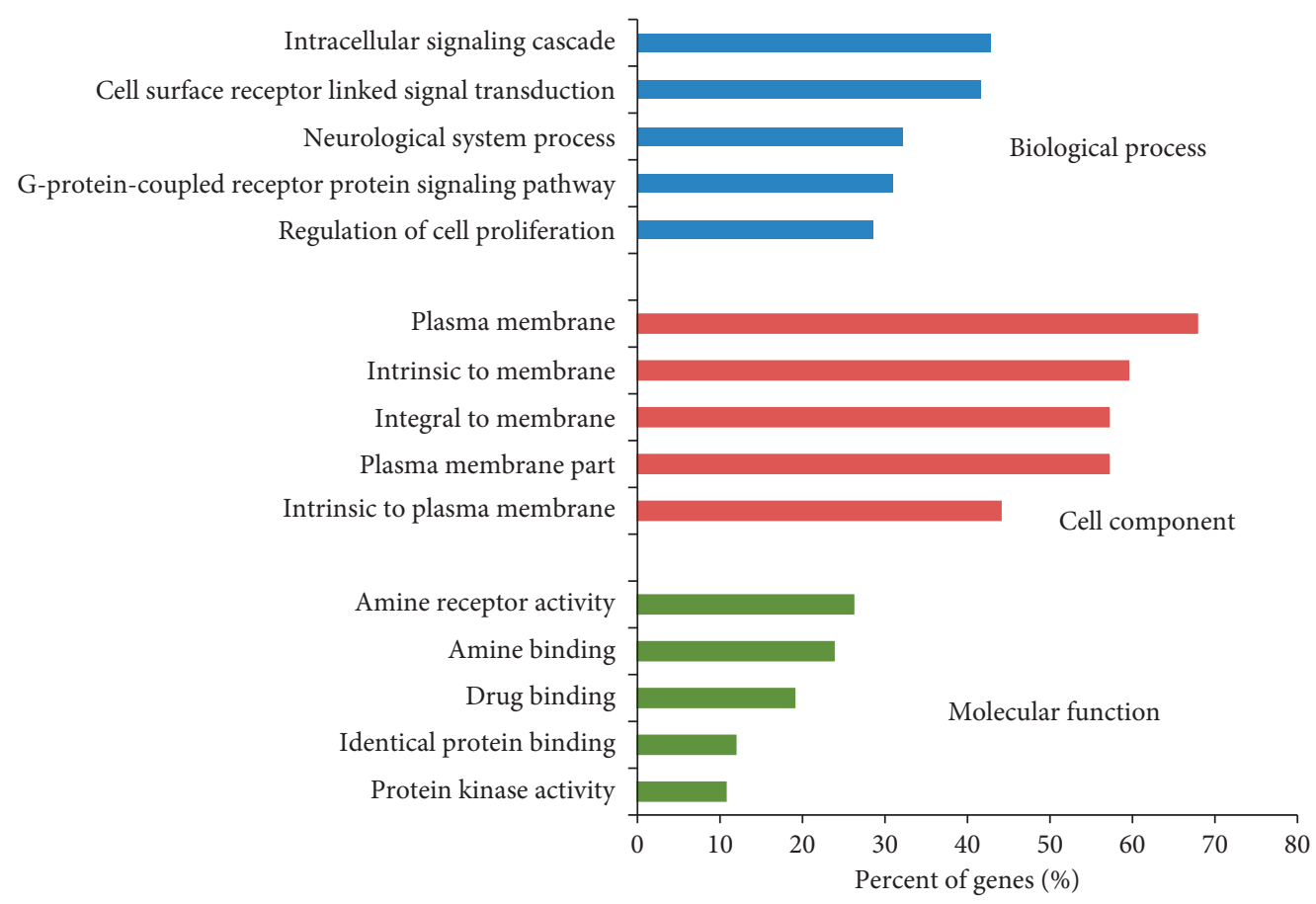

(a)

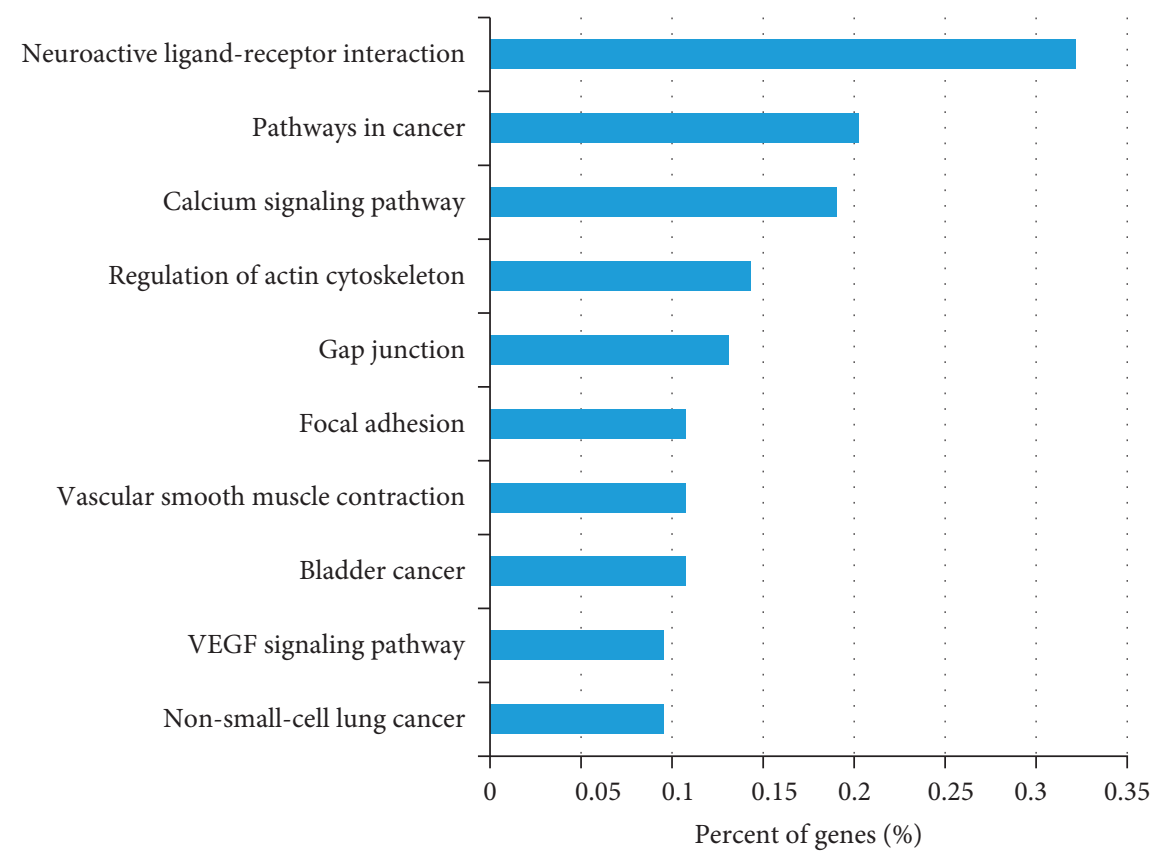

(b)

FIGURE 4: GO enrichment and pathway analysis of common targets. (a) Top 5 significantly enriched terms are shown in the biological process, cell component, and molecular function from DAVID. (b) The top 10 remarkably enriched pathways are shown.

system diseases (15.7\%), neoplasms (11.1\%), cardiovascular diseases $(11.1 \%)$, and pathological conditions, signs and symptoms (9.3\%). Among these diseases, female urogenital diseases and pregnancy complications contained $2.8 \%$ targets, mainly including EMS, estrogen deficiency, and cervical cancer. It was worthwhile to note that PTGS2 and MTOR were related to EMS. This suggests that JFS might have potential therapeutic effects on these diseases.
3.3. GO Enrichment and Pathway Analysis. In GO enrichment, 484 of 584 biological processes, 59 of 81 molecular functions, and 44 of 50 cell components were significantly identified in common targets from DAVID $(P<0.05)$ (Table S5). The top 5 remarkably enriched terms are listed in Figure 4(a). According to pathway analysis, 37 common target-related pathways were identified significantly in DAVID. The top 10 representative pathways were exhibited 
TABLE 2: Docking scores of JFS with apoptosis-related targets.

\begin{tabular}{|c|c|c|c|}
\hline Compound & Target & PDB ID & Docking score \\
\hline \multirow{6}{*}{ Ligustrazine } & Bax & $2 \mathrm{G} 5 \mathrm{~B}$ & 6.859 \\
\hline & PARP & 2RIQ & 6.641 \\
\hline & $\mathrm{Bcl}-2$ & $2 \mathrm{~W} 3 \mathrm{~L}$ & 5.938 \\
\hline & Caspase-3 & $2 \mathrm{DKO}$ & 5.105 \\
\hline & Caspase- 9 & 3D9T & 4.66 \\
\hline & $\mathrm{Bad}$ & 2BZW & - \\
\hline \multirow{6}{*}{ Ferulic acid } & Caspase-9 & 3D9T & 4.004 \\
\hline & Caspase-3 & 2DKO & 3.552 \\
\hline & Bcl-2 & 2W3L & 3.379 \\
\hline & $\mathrm{Bax}$ & $2 \mathrm{G} 5 \mathrm{~B}$ & 3.146 \\
\hline & PARP & 2RIQ & 3.05 \\
\hline & $\mathrm{Bad}$ & 2BZW & - \\
\hline \multirow{6}{*}{ Tetrahydropalmatine } & Caspase-9 & $3 \mathrm{D} 9 \mathrm{~T}$ & 3.84 \\
\hline & Caspase-3 & $2 \mathrm{DKO}$ & 3.755 \\
\hline & PARP & 2RIQ & 3.745 \\
\hline & Bcl-2 & $2 \mathrm{~W} 3 \mathrm{~L}$ & 3.709 \\
\hline & Bax & $2 \mathrm{G} 5 \mathrm{~B}$ & 3.701 \\
\hline & $\mathrm{Bad}$ & 2BZW & - \\
\hline
\end{tabular}

(Figure 4(b)). Bcl-2 was mainly distributed in 6 pathways, which were small-cell lung cancer, prostate cancer, pathways in cancer, neurotrophin signaling pathway, focal adhesion, and colorectal cancer (Table S5).

3.4. Binding Activity of JFS with Apoptosis-Related Targets. Descent of apoptosis in ectopic endometria is considered as the principal mechanism of EMS [19]. In Figure 2, Bcl-2 was one of the common targets between tetrahydropalmatine and drug targets. Consequently, apoptosis-related targets, including Bcl-2, Bax, Bad, caspase-3, caspase-9, and PARP, were prepared for molecular docking. Ligustrazine showed the binding activity with 5 targets, a certain binding with caspase- 9 (docking score $>4.25$ ) and the better binding with Bcl-2, Bax, and caspase-3, and PARP (docking score >5.0). Ferulic acid and tetrahydropalmatine had no obvious binding with 6 targets (Table 2). This indicates that ligustrazine in JFS may have a primary effect on apoptosis.

3.5. JFS Elevated the Reduction Rate of Volume in EMS. 28 days after surgery, EMS model was successfully demonstrated in 56 rats. The success rate of the model was $79 \%$. Difference in the ectopic endometrium volume was not obvious between 5 groups before administration. After treatment for 28 days, the volume change rate was measured in different groups compared with pretreatment. In the EMS group, the volume of ectopic endometrium was slightly reduced. The volume change rates of 90 and $180 \mathrm{mg} \cdot \mathrm{kg}^{-1} \mathrm{JFS}$ groups were obviously higher compared with the EMS group $(P<0.05)$ (Figure 5(a)). This suggests that JFS had obvious inhibitory effects on transplant growth in a dose-dependent manner.

3.6. JFS Degraded E2 and PROG Levels. EMS is considered as an estrogen-dependent disease, accompanied by perturbations in progesterone signal [20]. In the previous study, the therapeutic medicine for endometriosis has been based upon systemic hormonal alteration. So E2 and PROG are considered as the indicators of therapy $[21,22]$. The ELISA results showed that high serum levels of E2 and PROG were observed in the EMS group. The E2 and PROG levels in the JFS groups decreased compared with the EMS group, especially in 90 and $180 \mathrm{mg} \mathrm{kg}^{-1}$ JFS groups $(P<0.05)$ (Figures 5(b) and 5(c)).

3.7. JFS Promoted Apoptosis in Ectopic Endometrial Tissues. The red fluorescence intensity and apoptotic cells' number increased with the treatment of JFS (Figure 6(a)). Meanwhile, compared with the EMS group, the AI in endometrial tissues was obviously promoted by JFS, especially in $180 \mathrm{mg} \cdot \mathrm{kg}^{-1}$ JFS (Figure 6(b)). This suggests that JFS had induced apoptosis in ectopic endometrial tissues in a dosedependent manner.

3.8. Activation of Bcl-2 Pathway by JFS. Using JFS for 28 days, genes and proteins of Bcl-2 pathway were detected, including Bcl-2, Bax, Bad, caspase-3, caspase-9, and PARP. JFS decreased the mRNA level of Bcl-2 by raising the mRNA levels of Bax and Bad (Figures 7(a), 7(b), and 7(c)). JFS obviously upregulated the gene expression of caspase- 9 and caspase- 3 compared to the EMS group $(P<0.05)$ (Figures $7(\mathrm{~d})$ and $7(\mathrm{e})$ ). PARP mRNA level reduced in 45 and $90 \mathrm{mg} \cdot \mathrm{kg}^{-1}$ JFS groups (Figure $7(\mathrm{f})$ ).

Furthermore, the protein expression was in accordance with gene expression. The protein level of Bcl-2 attenuated in 90 and $180 \mathrm{mg} \cdot \mathrm{kg}^{-1}$ JFS groups, accompanied with accumulation of Bax (Figures 8(a), 8(b), and 8(c)). PARP protein decreased significantly in 45 and $90 \mathrm{mg} \cdot \mathrm{kg}^{-1} \mathrm{JFS}$ groups and however increased in $180 \mathrm{mg} \cdot \mathrm{kg}^{-1}$ JFS group. It was noteworthy that the protein expression of cleaved-PARP remarkably enhanced in $180 \mathrm{mg} \cdot \mathrm{kg}^{-1} \mathrm{JFS}$ group compared with the EMS group $(P<0.05)$ (Figures $8(\mathrm{a}), 8(\mathrm{~d})$, and $8(\mathrm{e}))$. 


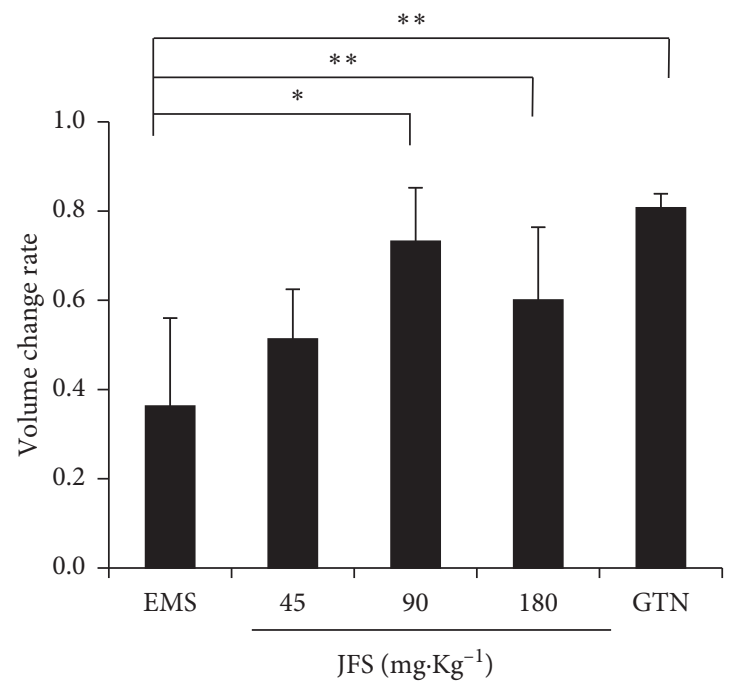

(a)

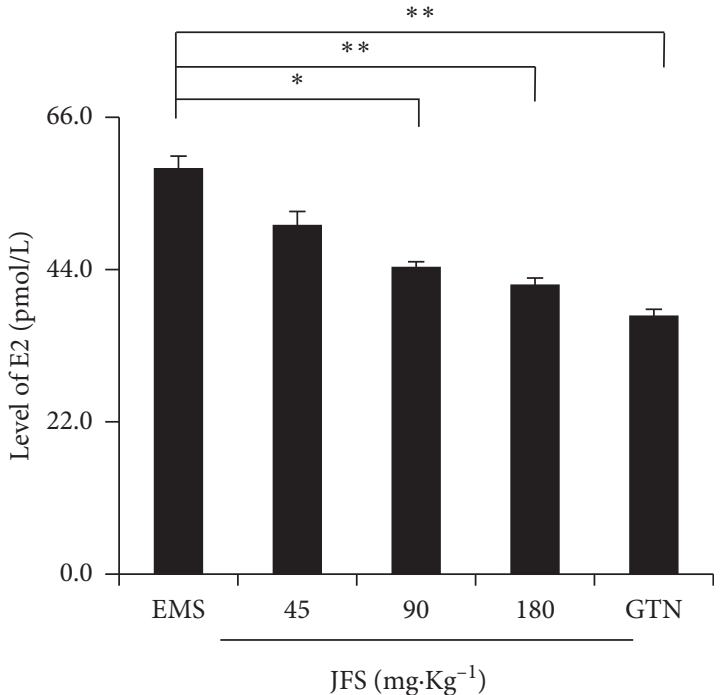

(b)

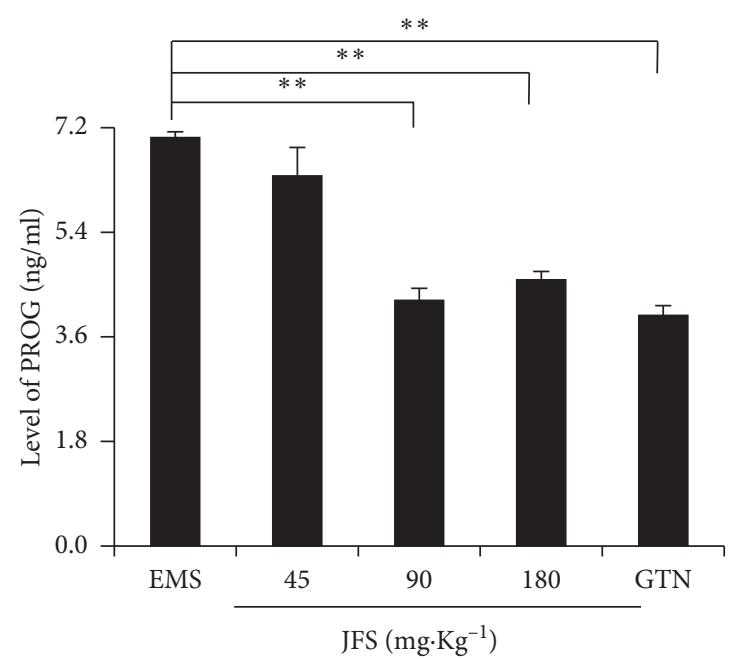

(c)

FIGURE 5: Regulation of JFS on volume change rate of ectopic endometrium and the levels of E2 and PROG. (a) The volume change rates of ectopic endometrium were calculated after administration for 28 days. (b)-(c) Serum levels of E2 and PROG were detected by ELISA in different groups. ${ }^{*} P<0.05,{ }^{* *} P<0.01$ compared with the EMS group. Columns, mean $(n=6)$. Bars, SD. EMS, endometriosis; JFS, Jiawei Foshou San; GTN, gestrinone.

This result suggests that the activation of apoptosis by JFS was connected with promotion of Bcl-2 pathway.

\section{Discussion}

Systems pharmacology is an ideal method to study the interaction between multicomponents, multitargets, and different pathways from online databases. Nevertheless, not all compound targets are pharmacological drug targets. So it will be more efficient to combine the systems pharmacology databases with therapeutic FDA-approved drug targets. Following this screening strategy, PTGS2 and MTOR were discovered as EMS drug targets associated with tetrahydropalmatine, one of the JFS components. Although ligustrazine and ferulic acid suppressed PTGS2 and MTOR in tumor or nervous system diseases [23-27], the cooperation of ligustrazine, ferulic acid, and tetrahydropalmatine is unknown whether or not via PTGS2 or MTOR, in spite of their valid effect in EMS. It needs further research. At the same time, it was worthwhile to note that 6 common targets were inspected among ferulic acid, tetrahydropalmatine, and drug targets, which comprise ADRB2, CA2, F3, PTGS1, SLC6A2, and SLC6A3. ADRB2 encodes beta-2-adrenergic receptor which mainly binds epinephrine. It is reported that activation of ADRB2 promotes EMS development by stress and surgery [28-30]. In cancer and cardiomyocytes, ADRB2 inhibits apoptosis mainly through $\mathrm{Bcl}-2 / \mathrm{Bax} /$ caspase-9 pathway [31-35]. Herein, ADRB2 might be the therapeutic target in EMS. We should try to use ADRB2 inhibitor in EMS or screen TCM drugs on ADRB2. 


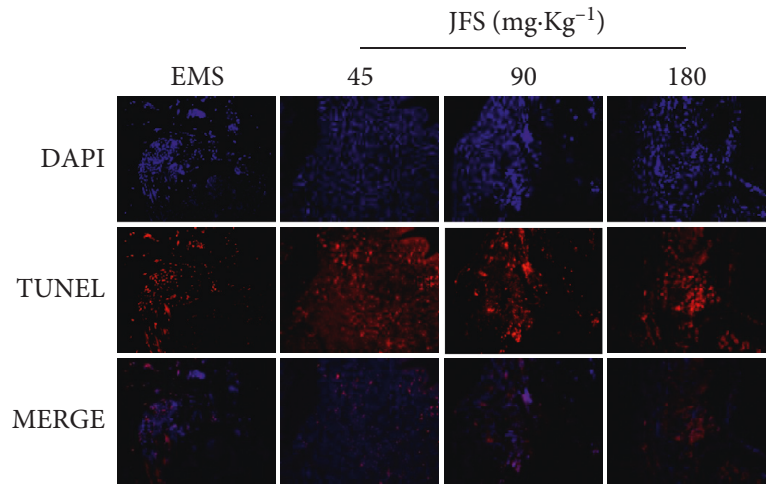

(a)

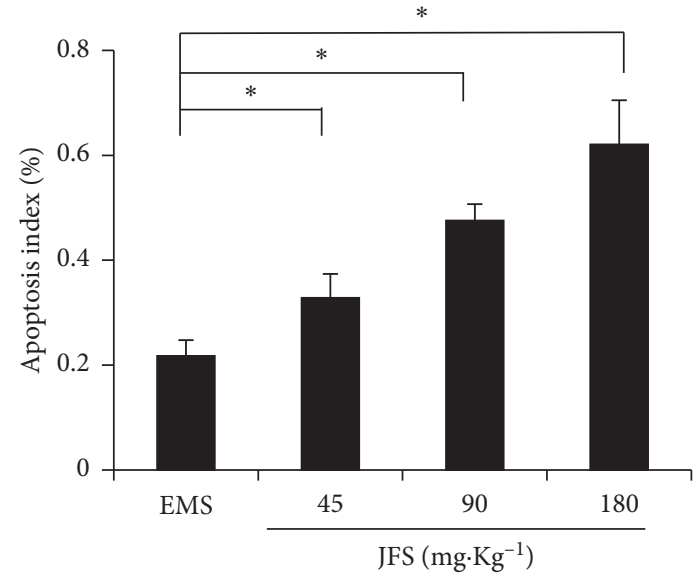

(b)

Figure 6: Detection of apoptosis in ectopic endometrial tissues. (a) Apoptosis in ectopic endometria of different groups was observed by TUNEL assay. DAPI-stained nuclei appeared in blue. Red-stained tissue appeared in red due to the presence of apoptotic cells. (b) The apoptotic index of ectopic endometrial tissues. ${ }^{*} P<0.05$ compared to the EMS group. Columns, mean $(n=3)$. Bars, SD. Magnification, $\times 200$. EMS, endometriosis; JFS, Jiawei Foshou San.

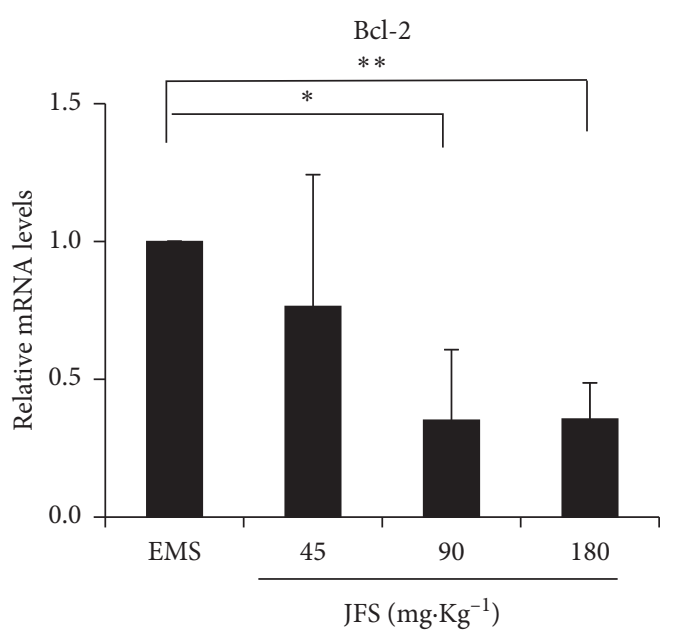

(a)

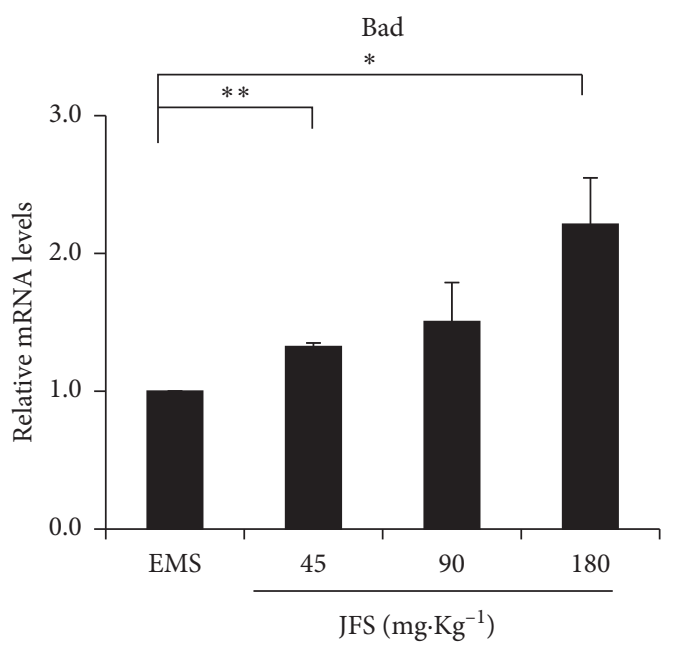

(c)

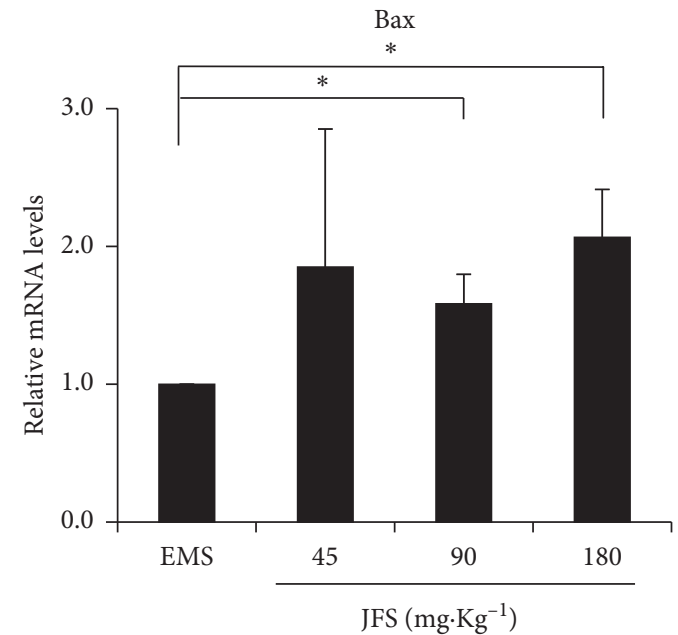

(b)

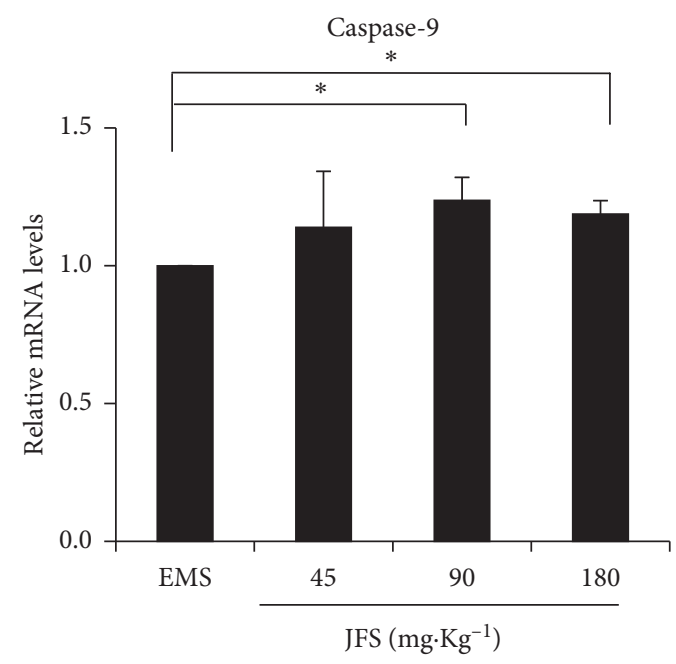

(d)

Figure 7: Continued. 


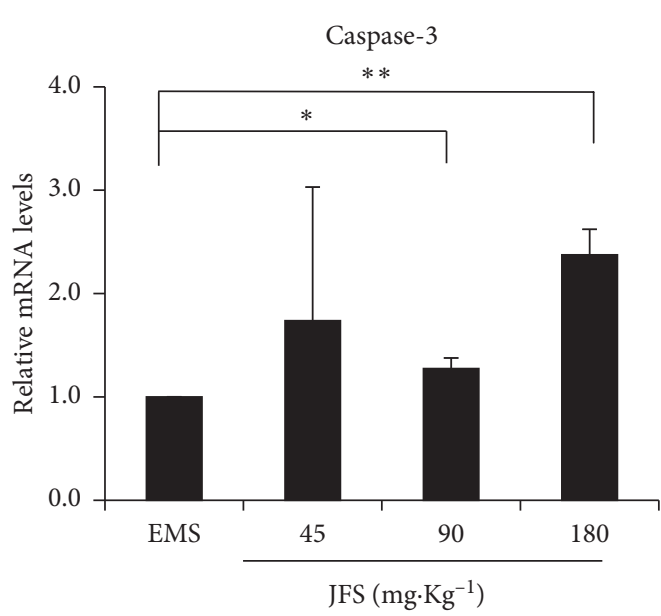

(e)

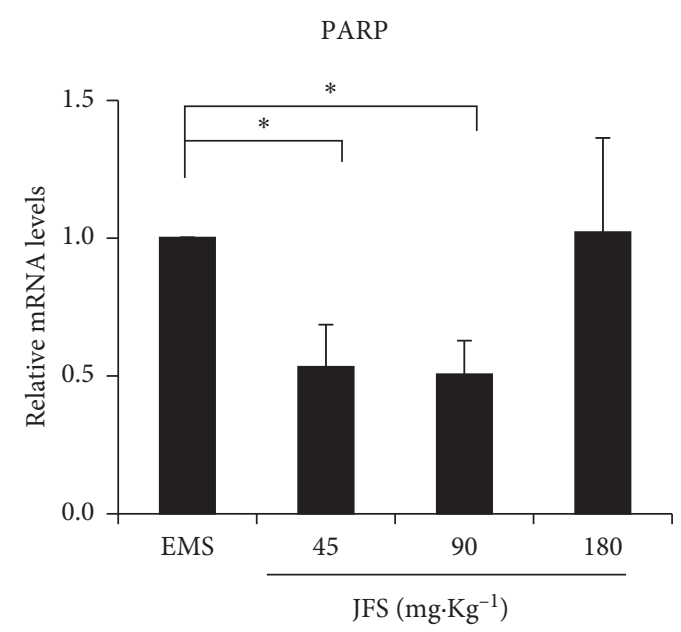

(f)

Figure 7: Gene expression of Bcl-2 pathway by JFS. (a)-(f) The mRNA levels of Bcl-2, Bax, Bad, caspase-3, caspase-9, and PARP were detected by qPCR in different groups. ${ }^{*} P<0.05$ compared to the EMS group. Columns, mean $(n=3)$. Bars, SD. EMS, endometriosis; JFS, Jiawei Foshou San.

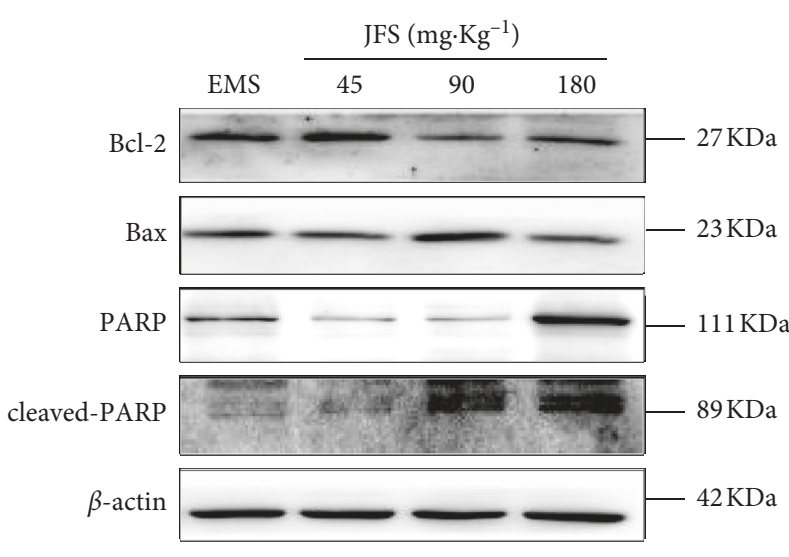

(a)

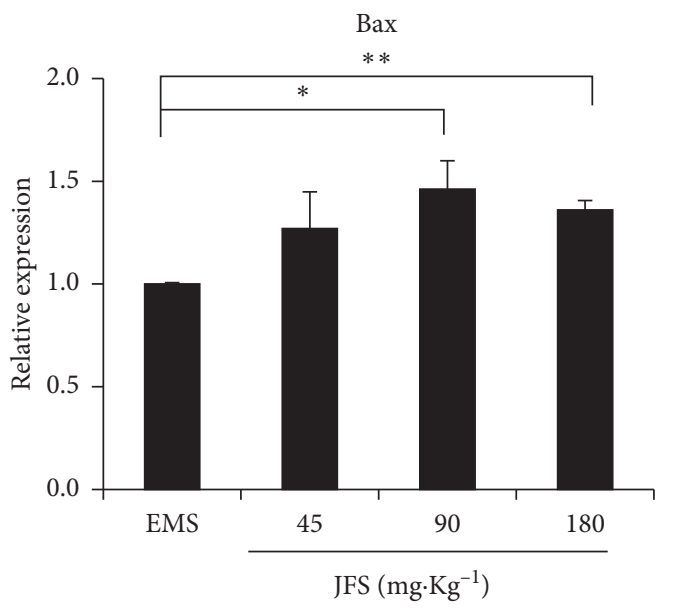

(c)

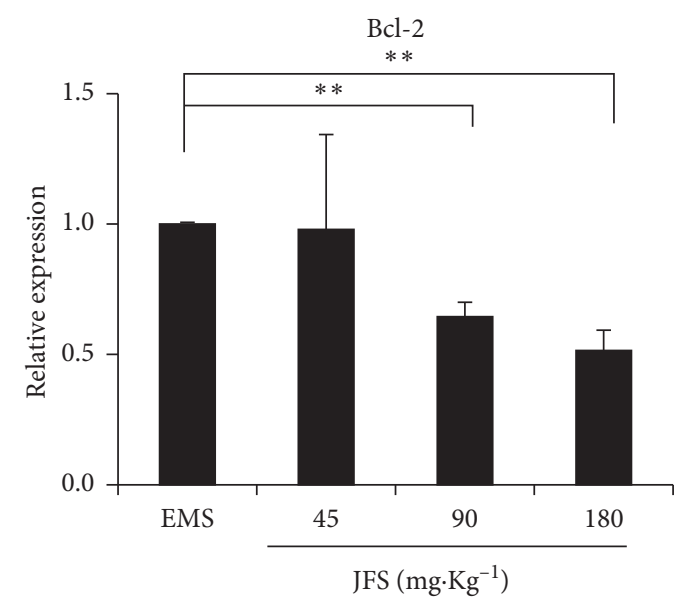

(b)

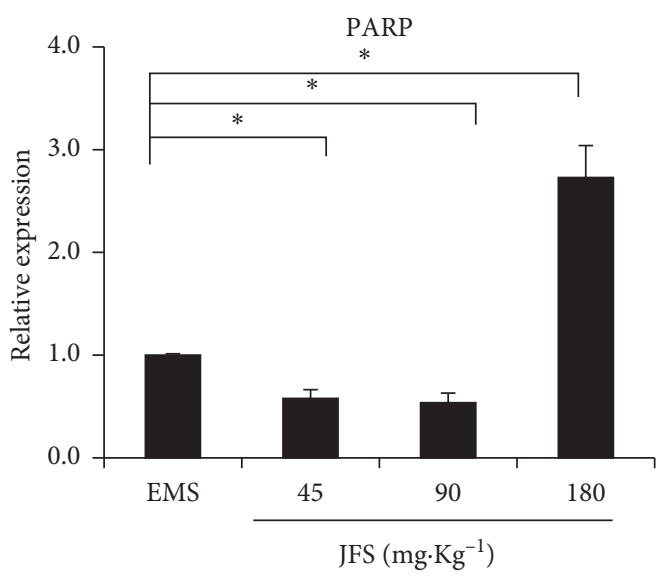

(d)

Figure 8: Continued. 


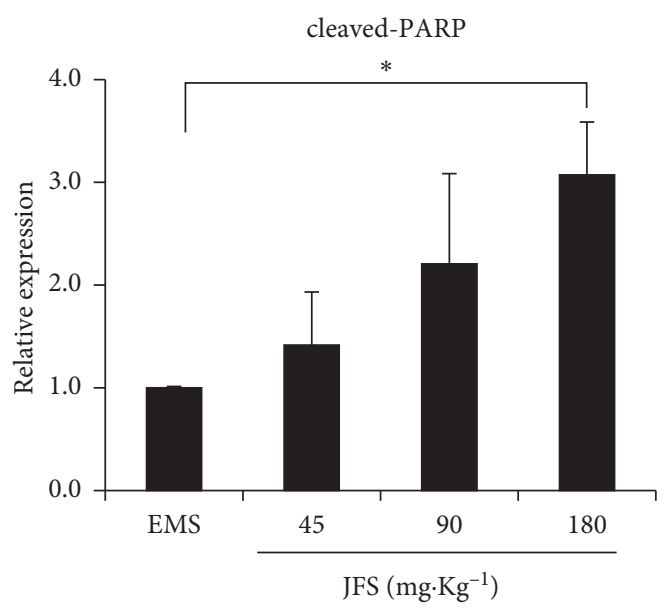

(e)

Figure 8: Protein levels of Bcl-2 pathway treated with JFS. (a)-(e) The protein levels of Bcl-2, Bax, PARP, and cleaved-PARP were detected by western blotting, and the ratios of Bcl-2, Bax, PARP, and cleaved-PARP to $\beta$-actin are shown. ${ }^{*} P<0.05$ compared to EMS, ${ }^{* *} P<0.01$ compared to EMS. Columns, mean $(n=3)$. Bars, SD. EMS, endometriosis; JFS, Jiawei Foshou San.

In JFS target-drug target network, Bcl-2 was the target of tetrahydropalmatine. But in molecular docking, docking score between tetrahydropalmatine and Bcl-2 was 3.709, and docking score between ligustrazine and Bcl-2 was 5.938 . Those implied that ligustrazine not tetrahydropalmatine showed the binding with $\mathrm{Bcl}-2$. In recent years, ligustrazine and tetrahydropalmatine have been found different effect on apoptosis through $\mathrm{Bcl}-2$ pathway. On the one hand, tetrahydropalmatine reduces the apoptosis through enhancing the expression of $\mathrm{Bcl}-2$ and reducing $\mathrm{Bax} / \mathrm{Bcl}-2$ ratio in cerebral ischemia-reperfusion and endothelial cells against $\gamma$-irradiation injury [36-38]. On the other hand, levo-tetrahydropalmatine induces apoptosis through upregulating $\mathrm{Bcl}-2$ in hepatocytes [39]. Meanwhile, ligustrazine exerts the opposite regulation of Bcl-2, including inhibiting apoptosis in acute myocardial ischemia and neuron cells, triggering apoptosis in cancer [40-43]. Basically, the perplexing effect of ligustrazine and tetrahydropalmatine on apoptosis might relate to the concentration and pathological environments in various diseases.

Bcl-2 is a well-known regulator inhibiting cellular apoptosis, while Bax and Bad trigger the cascades leading to apoptosis. Downstream caspase-9 causes activation of caspase-3, which induces PARP to cleavage PARP [44]. In this study, JFS elevated apoptosis of ectopic endometrium using the TUNEL assay in the EMS rat model. In addition, the gene and protein expression of $\mathrm{Bcl}-2$ diminished with rising Bax and Bad by JFS. Caspase- 9 and caspase- 3 genes expressed much more than the EMS group. JFS promoted the protein level of cleaved-PARP. It is noticeable that $180 \mathrm{mg} \cdot \mathrm{kg}^{-1} \mathrm{JFS}$ enhanced the PARP, while increasing cleaved-PARP. In the previous study, JFS ingredients have different effects on PARP, tetrahydropalmatine, and ferulic acid attenuating it $[38,45-49]$ and ligustrazine promoting it [50]. So it might be the foundation of the synergistic therapeutic effect of JFS ingredients. The above data suggest that apoptotic promotion of JFS may be related to Bcl-2 pathway in EMS. But in the future, the effect of JFS on other apoptosis factors such as P53, cyt C, caspase-6, and caspase-7 needs further investigation.

\section{Conclusions}

In this study, using systems pharmacology databases, the connection was uncovered between JFS ingredients, JFS targets, FDA-approved drug targets, disease, and pathways. In molecular docking, JFS ingredients were bound to apoptosis-related targets. JFS promoted apoptosis through activation of Bcl-2 pathway in EMS.

\section{Data Availability}

The network, GO, and pathway analysis data used to support the findings of this study are included within the supplementary information files. The other data used to support the findings of this study are available from the corresponding author upon request.

\section{Conflicts of Interest}

The authors declare that there are no conflicts of interest regarding the publication of this paper.

\section{Authors' Contributions}

Jiahui Wei and Binxin Zhao contributed equally to this work.

\section{Acknowledgments}

This work was supported by the National Natural Science Foundation of China (nos. 81773984 and 81402441); Traditional Chinese Medicine Research Project of Chongqing Health Bureau (no. zy201602125); Southwest University National Undergraduate Training Program for Innovation 
and Entrepreneurship (no. 201710635073); and SWU National Experimental Demonstration Center of Pharmacy (nos. XY2017-CXZD-04 and YX2017-CXYB-01).

\section{Supplementary Materials}

Table S1: detailed information on targets for JFS compounds. Table S2: detailed information on pharmacological targets of FDA-approved drugs. Table S3: 99 common targets between JFS targets and FDA-approved drug targets. Table S4: detailed information on common target-diseases and disease classification. Table S5: GO analysis and KEGG pathway enrichment of 99 common targets from DAVID. (Supplementary Materials)

\section{References}

[1] F. Liao, "Herbs of activating blood circulation to remove blood stasis," Clinical Hemorheology and Microcirculation, vol. 23, no. 2-4, pp. 127-131, 2000.

[2] G. Yu and J. Wang, "Blood stasis syndrome of coronary heart disease: a perspective of modern medicine," Chinese Journal of Integrative Medicine, vol. 20, no. 4, pp. 300-306, 2014.

[3] Y. Chen, J. Wei, Y. Zhang et al., "Anti-endometriosis mechanism of Jiawei Foshou san based on network pharmacology," Frontiers in Pharmacology, vol. 9, p. 811, 2018.

[4] G. H. Zheng, S. Q. Xiong, H. Y. Chen, L. J. Mei, and T. Wang, "Association of platelet-activating factor receptor gene rs5938 $(\mathrm{G} / \mathrm{T})$ and rs313152 (T/C) polymorphisms with coronary heart disease and blood stasis syndrome in a Chinese Han population," Chinese Journal of Integrative Medicine, vol. 23, no. 12, pp. 893-900, 2017.

[5] H. Bai, P. Q. Li, J. Liu, and X. P. Liu, "Association analysis on traditional efficacy and modern research of Foshou San," Chinese Traditional Patent Medicine, vol. 36, no. 3, pp. 601604, 2014.

[6] Q. Tang, F. H. Shang, X. C. Wang et al., "Combination use of ferulic acid, ligustrazine and tetrahydropalmatine inhibits the growth of ectopic endometrial tissue: a multi-target therapy for endometriosis rats," Journal of Ethnopharmacology, vol. 151, no. 3, pp. 1218-1225, 2014.

[7] R. Zhang, X. Zhu, H. Bai, and K. Ning, "Network pharmacology databases for traditional Chinese medicine: review and assessment," Frontiers in Pharmacology, vol. 10, p. 123, 2019.

[8] X. Q. Shi, S. J. Yue, Y. P. Tang et al., "A network pharmacology approach to investigate the blood enriching mechanism of Danggui buxue Decoction," Journal of Ethnopharmacology, vol. 235, pp. 227-242, 2019.

[9] M. Rask-Andersen, M. S. Almen, and H. B. Schioth, "Trends in the exploitation of novel drug targets," Nature Reviews Drug Discovery, vol. 10, no. 8, pp. 579-590, 2011.

[10] X. Ma, Y. Hui, L. Lin, Y. Wu, X. Zhang, and X. Qin, "Possible relevance of tumor-related genes mutation to malignant transformation of endometriosis," European Journal of Gynaecological Oncology, vol. 37, no. 1, pp. 89-94, 2016.

[11] A. Mihailovici, M. Rottenstreich, S. Kovel, I. Wassermann, N. Smorgick, and Z. Vaknin, "Endometriosis-associated malignant transformation in abdominal surgical scar: a PRISMA-compliant systematic review," Medicine, vol. 96, no. 49, Article ID e9136, 2017.

[12] V. A. Gomes, C. M. Bonocher, E. S. J. C. Rosa, C. C. P. de Paz, R. A. Ferriani, and J. Meola, "The Apoptotic, Angiogenic and Cell Proliferation Genes CD63, S100A6 e GNB2L1 are Altered in Patients with Endometriosis," Revista Brasileira de Ginecologia e Obstetrícia, vol. 40, no. 10, pp. 606-613, 2018.

[13] A. M. Dull, M. A. Moga, O. G. Dimienescu, G. Sechel, V. Burtea, and C. V. Anastasiu, "Therapeutic approaches of resveratrol on endometriosis via anti-inflammatory and antiangiogenic pathways," Molecules, vol. 24, no. 4, p. 667, 2019.

[14] K. Y. Hsin, S. Ghosh, and H. Kitano, "Combining machine learning systems and multiple docking simulation packages to improve docking prediction reliability for network pharmacology," PLoS One, vol. 8, no. 12, Article ID e83922, 2013.

[15] Z. Liu, F. Guo, Y. Wang et al., "BATMAN-TCM: a bioinformatics analysis tool for molecular mechANism of traditional Chinese medicine," Scientific Reports, vol. 6, no. 1, Article ID 21146, 2016.

[16] N. Pinar, O. S. Karapinar, O. Ozcan, T. Ozgur, and S. Bayraktar, "Effect of alpha-lipoic acid on endometrial implants in an experimental rat model," Fundamental \& Clinical Pharmacology, vol. 31, no. 5, pp. 506-512, 2017.

[17] H. Lv, J. Li, and Y. Q. Che, "CXCL8 gene silencing promotes neuroglial cells activation while inhibiting neuroinflammation through the PI3K/Akt/NF-kappaB-signaling pathway in mice with ischemic stroke," Journal of Cellular Physiology, vol. 234, no. 5, pp. 7341-7355, 2019.

[18] Y. Chen, D. K. Yu, C. X. Zhang et al., "Lidamycin inhibits tumor initiating cells of hepatocellular carcinoma Huh7 through GSK3 beta/beta-Catenin pathway," Molecular Carcinogenesis, vol. 54, no. 1, pp. 1-8, 2015.

[19] S. J. Han, S. Y. Jung, S.-P. Wu et al., "Estrogen receptor beta modulates apoptosis complexes and the inflammasome to drive the pathogenesis of endometriosis," Cell, vol. 163, no. 4, pp. 960-974, 2015.

[20] P. Vercellini, P. Vigano, E. Somigliana, and L. Fedele, "Endometriosis: pathogenesis and treatment," Nature Reviews Endocrinology, vol. 10, no. 5, pp. 261-275, 2014.

[21] M. A. Bedaiwy, C. Allaire, and S. Alfaraj, "Long-term medical management of endometriosis with dienogest and with a gonadotropin-releasing hormone agonist and add-back hormone therapy," Fertility and Sterility, vol. 107, no. 3, pp. 537-548, 2017.

[22] B. Gurates and S. E. Bulun, "Endometriosis: the ultimate hormonal disease," Seminars in Reproductive Medicine, vol. 21, no. 2, pp. 125-134, 2003.

[23] C. Y. Cheng, S. T. Kao, and Y. C. Lee, "Ferulic acid ameliorates cerebral infarction by activating Akt/mTOR/4EBP1/Bcl2 antiapoptotic signaling in the penumbral cortex following permanent cerebral ischemia in rats," Molecular Medicine Reports, vol. 19, no. 2, pp. 792-804, 2018.

[24] B. W. Kim, S. Koppula, S. Y. Park et al., "Attenuation of neuroinflammatory responses and behavioral deficits by Ligusticum officinale (Makino) Kitag in stimulated microglia and MPTP-induced mouse model of Parkinson's disease," Journal of Ethnopharmacology, vol. 164, pp. 388-397, 2015.

[25] C. Mancuso and R. Santangelo, "Ferulic acid: pharmacological and toxicological aspects," Food and Chemical Toxicology, vol. 65, pp. 185-195, 2014.

[26] H. E. Michel, M. G. Tadros, A. Esmat, A. E. Khalifa, and A. M. Abdel-Tawab, "Tetramethylpyrazine ameliorates rotenone-induced Parkinson's disease in rats: involvement of its anti-inflammatory and anti-apoptotic actions," Molecular Neurobiology, vol. 54, no. 7, pp. 4866-4878, 2017.

[27] B. Yu, M. Ruan, T. Liang et al., "Tetramethylpyrazine phosphate and borneol combination therapy synergistically attenuated ischemia-reperfusion injury of the hypothalamus and striatum via regulation of apoptosis and autophagy in a 
rat model," American Journal of Translational Research, vol. 9, no. 11, pp. 4807-4820, 2017.

[28] S. W. Guo, Q. Zhang, and X. Liu, "Social psychogenic stress promotes the development of endometriosis in mouse," Reproductive BioMedicine Online, vol. 34, no. 3, pp. 225-239, 2017.

[29] Q. Long, X. Liu, and S. W. Guo, "Surgery accelerates the development of endometriosis in mice," American Journal of Obstetrics and Gynecology, vol. 215, no. 3, pp. 320 e321-320 e315, 2016.

[30] Q. Long, X. Liu, Q. Qi, and S. W. Guo, "Chronic stress accelerates the development of endometriosis in mouse through adrenergic receptor beta2," Human Reproduction, vol. 31, no. 11, pp. 2506-2519, 2016.

[31] Y. Kang, A. S. Nagaraja, G. N. Armaiz-Pena et al., "Adrenergic stimulation of DUSP1 impairs chemotherapy response in ovarian cancer," Clinical Cancer Research, vol. 22, no. 7, pp. 1713-1724, 2016.

[32] X. Yang, T. Zhao, L. Feng et al., "PM2.5-induced ADRB2 hypermethylation contributed to cardiac dysfunction through cardiomyocytes apoptosis via PI3K/Akt pathway," Environment International, vol. 127, pp. 601-614, 2019.

[33] S. Hassan, Y. Karpova, D. Baiz et al., "Behavioral stress accelerates prostate cancer development in mice," Journal of Clinical Investigation, vol. 123, no. 2, pp. 874-886, 2013.

[34] G. Kulik, "ADRB2-Targeting therapies for prostate cancer," Cancers, vol. 11, no. 3, p. 358, 2019.

[35] D. Dang, J. Zhang, and J. Yang, "Growth inhibition of human hepatocellular carcinoma cells by antagonism of the beta2 adrenergic receptor," Oncology Letters, vol. 16, no. 2, pp. 1425-1430, 2018.

[36] J. Yu, B. K. Piao, Y. X. Pei, X. Qi, and B. J. Hua, "Protective effects of tetrahydropalmatine against gamma-radiation induced damage to human endothelial cells," Life Sci, vol. 87, no. 1-2, pp. 55-63, 2010.

[37] B. Liu and G. Yang, "Effects of L-tetrahydropalmatine on the expressions of bcl-2 and bax in rat after acute global cerebral ischemia and reperfusion," Journal of Huazhong University of Science and Technology (Medical Sciences), vol. 24, no. 5, pp. 445-448, 2004.

[38] R. Sun, Y. Song, S. Li et al., "Levo-tetrahydropalmatine attenuates neuron apoptosis induced by cerebral ischemiareperfusion injury: involvement of c-abl activation," Journal of Molecular Neuroscience, vol. 65, no. 3, pp. 391-399, 2018.

[39] C. Wang, J. Zhou, S. Wang et al., "Shotgun approach based comparative proteomic analysis of levo-tetrahydropalmatineinduced apoptosis in hepatocytes," Toxicology Letters, vol. 194, no. 1-2, pp. 8-15, 2010.

[40] T. Zhao, Y. Fu, H. Sun, and X. Liu, "Ligustrazine suppresses neuron apoptosis via the $\mathrm{Bax} / \mathrm{Bcl}-2$ and caspase- 3 pathway in PC12 cells and in rats with vascular dementia," IUBMB Life, vol. 70, no. 1, pp. 60-70, 2018.

[41] Q. Yang, D. D. Huang, D. G. Li et al., "Tetramethylpyrazine exerts a protective effect against injury from acute myocardial ischemia by regulating the PI3K/Akt/GSK-3beta signaling pathway," Cellular \& Molecular Biology Letters, vol. 24, no. 1, p. $17,2019$.

[42] H. H. Huang, F. B. Liu, Z. Ruan, J. Zheng, Y. J. Su, and J. Wang, "Tetramethylpyrazine (TMPZ) triggers S-phase arrest and mitochondria-dependent apoptosis in lung cancer cells," Neoplasma, vol. 65, no. 3, pp. 367-375, 2018.

[43] S. Bi, F. Chu, M. Wang et al., "Ligustrazine-oleanolic acid Glycine derivative, G-TOA, selectively inhibited the proliferation and induced apoptosis of activated HSC-T6 cells," Molecules, vol. 21, no. 11, p. 1599, 2016.

[44] F. Bina, S. Soleymani, T. Toliat et al., "Plant-derived medicines for treatment of endometriosis: a comprehensive review of molecular mechanisms," Pharmacological Research, vol. 139, pp. 76-90, 2019.

[45] S. Chowdhury, S. Ghosh, K. Rashid, and P. C. Sil, "Deciphering the role of ferulic acid against streptozotocin-induced cellular stress in the cardiac tissue of diabetic rats," Food and Chemical Toxicology, vol. 97, pp. 187-198, 2016.

[46] U. Das, A. Sengupta, S. Biswas et al., "Alteration of murine duodenal morphology and redox signalling events by reactive oxygen species generated after whole body gamma-irradiation and its prevention by ferulic acid," Free Radical Research, vol. 51, no. 11-12, pp. 886-910, 2017.

[47] S. Ghosh, S. Chowdhury, P. Sarkar, and P. C. Sil, "Ameliorative role of ferulic acid against diabetes associated oxidative stress induced spleen damage," Food and Chemical Toxicology, vol. 118, pp. 272-286, 2018.

[48] U. Fahrioglu, Y. Dodurga, L. Elmas, and M. Secme, "Ferulic acid decreases cell viability and colony formation while inhibiting migration of MIA PaCa-2 human pancreatic cancer cells in vitro," Gene, vol. 576, no. 1, pp. 476-482, 2016.

[49] F. Di Domenico, M. Perluigi, C. Foppoli et al., "Protective effect of ferulic acid ethyl ester against oxidative stress mediated by UVB irradiation in human epidermal melanocytes," Free Radical Research, vol. 43, no. 4, pp. 365-375, 2009.

[50] J. Cao, Q. Miao, S. Miao et al., “Tetramethylpyrazine (TMP) exerts antitumor effects by inducing apoptosis and autophagy in hepatocellular carcinoma," International Immunopharmacology, vol. 26, no. 1, pp. 212-220, 2015. 


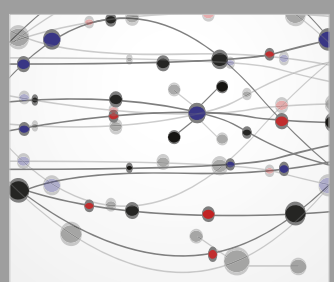

The Scientific World Journal
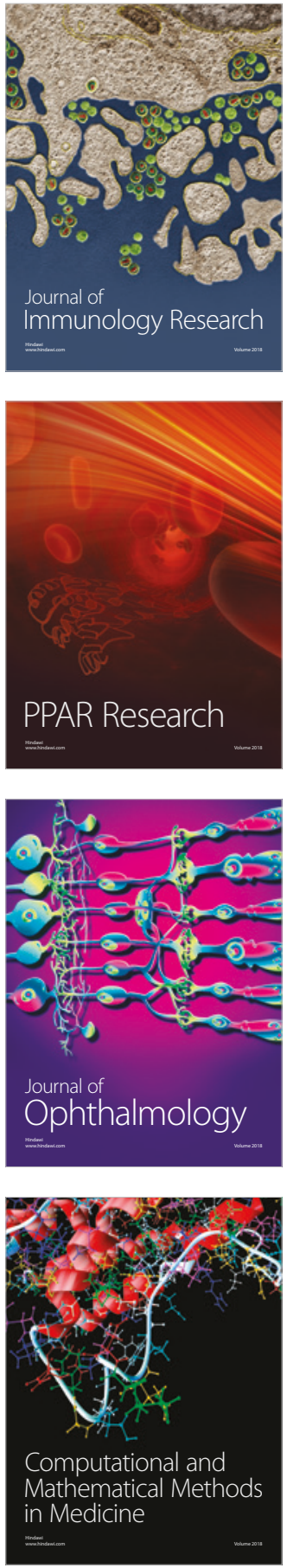

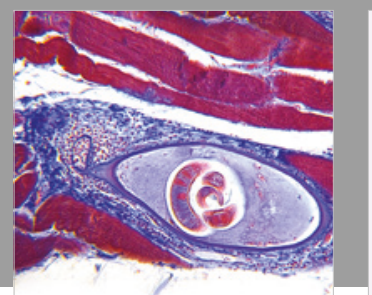

Gastroenterology Research and Practice

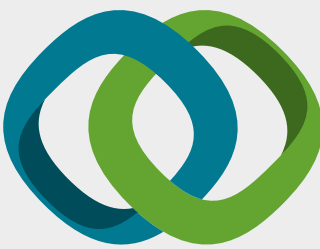

\section{Hindawi}

Submit your manuscripts at

www.hindawi.com
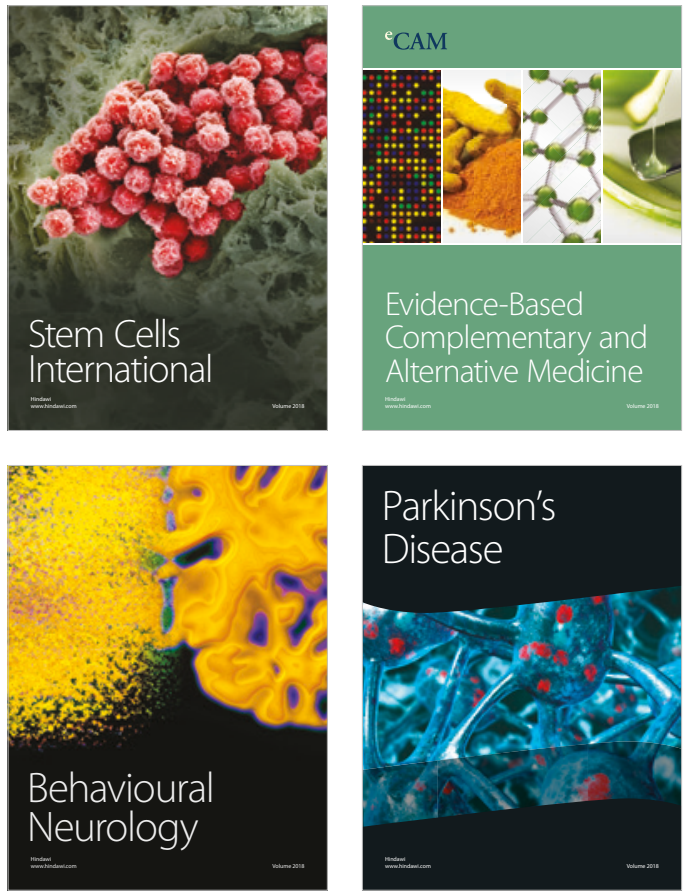

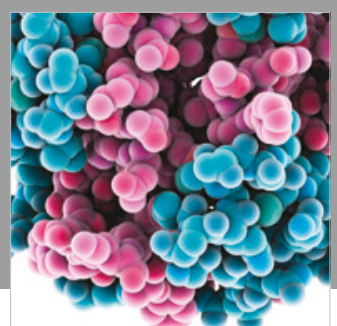

ournal of

Diabetes Research

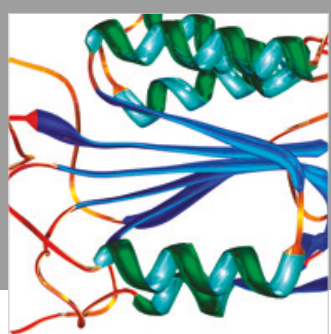

Disease Markers
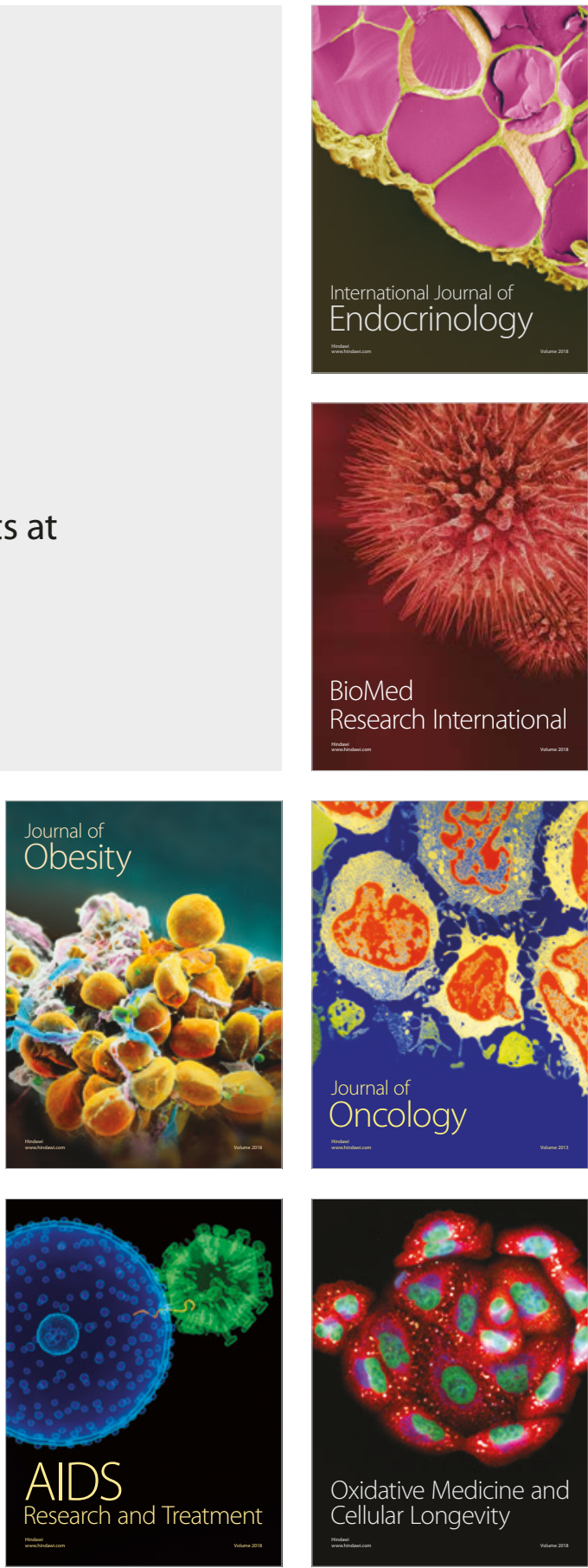